\title{
Effect of serum triglyceride level on the prognosis of patients with hepatocellular carcinoma in the absence of cirrhosis
}

\author{
Xiaoli Liu, Mengge Li, Xinhui Wang, Zhibo Dang, Yuyong Jiang, Xianbo Wang and Zhiyun Yang*
}

\begin{abstract}
Background: The liver plays an important role in the metabolism of lipid and lipoprotein. Dyslipidemia has been demonstrated to be related with several cancers, but the association between serum lipid and hepatocellular carcinoma (HCC) in the absence of cirrhosis remains unclear.

Methods: A total of 2528 patients with HCC at the Beijing Ditan Hospital between February 2008 and December 2017 were retrospectively included in the study. We identified 200 patients with HCC without cirrhosis by histopathology, imaging, endoscopic findings, and laboratory tests. Multivariate regression analysis was performed to determine the independent characteristics associated with HCC without cirrhosis and its prognosis.

Results: In the logistics regression analysis, compared to patients with HCC with cirrhosis, patients with HCC without cirrhosis were more likely to have elevated triglyceride (TG) levels $(\mathrm{OR}=2.66 ; 95 \% \mathrm{Cl}, 1.18-6.01 ; P=0.019)$. The Kaplan-Meier analysis revealed that a lower TG level was a risk factor regardless of the presence of cirrhosis. The results of the Cox proportional hazard regression analysis showed that a decreased TG level was significantly related to a worse overall survival $(H R=0.51 ; 95 \% \mathrm{Cl}, 0.29-0.89 ; P=0.017)$.

Conclusion: Serum TG level may be an independent factor to predict the prognosis of patients with HCC in the absence of cirrhosis.
\end{abstract}

Keywords: TG, HCC, Lipid, Lipoprotein, Prognosis

\section{Introduction}

Hepatocellular carcinoma ( $\mathrm{HCC})$ is the fifth most common malignancy and has the second highest cancer mortality worldwide.[1, 2]. Although most HCCs are usually accompanied by cirrhosis owing to chronic viral infections, a certain number of patients with HCC, ranging widely from 7 to $54 \%$, do not have cirrhosis[3-6]. It is perceived that the liver is one of the most important organs in multiple metabolite pathways[7], including lipid and lipoprotein. Hepatic cellular necrosis caused by cirrhosis and HCC leads to aberrations in serum lipid and lipoprotein levels[8,9]. Some studies reported that the triglyceride (TG) level significantly decreased in patients with cirrhosis or HCC[10-12]. However, the

\footnotetext{
* Correspondence: yangzhiyun2016@163.com

Center for Integrative Medicine, Beijing Ditan Hospital, Capital Medical University, No. 8 Jing Shun East Street, Beijing 100015, People's Republic of China
}

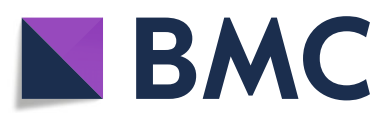

(c) The Author(s). 2018 Open Access This article is distributed under the terms of the Creative Commons Attribution 4.0 International License (http://creativecommons.org/licenses/by/4.0/), which permits unrestricted use, distribution, and reproduction in any medium, provided you give appropriate credit to the original author(s) and the source, provide a link to the Creative Commons license, and indicate if changes were made. The Creative Commons Public Domain Dedication waiver (http://creativecommons.org/publicdomain/zero/1.0/) applies to the data made available in this article, unless otherwise stated. with HCC without cirrhosis remain unclear.

Recently, abnormal lipid and lipoprotein levels were considered to be related with the incidence and development of several types of cancer[13, 14]. Several researches showed that elevated TG level and suppressed high-density lipoprotein cholesterol (HDLc) level were related to a high risk of occurrence and death in colon, breast, lung, and prostate cancers[15-18]. However, the relationships between blood profile and liver cancer were contradictory due to the complex etiology. Several studies that considered the risk factor of non-alcoholic fatty liver disease (NAFLD) revealed that elevated TG and low HDLc levels attribute to a greater risk of HCC in patients with cirrhosis[19]. In contrast, regarding HBV and $\mathrm{HCV}$ infection, TG levels were inverse contributory factors for $\mathrm{HCC}[20,21]$. Furthermore, the prognostic effect of lipid profiles on HCC remains unclear. Few 
studies indicated that low cholesterol and HDLc levels could predict the recurrence of HCC in patients after liver resections[22]. However, whether the alterations of lipid profiles are correlated with HCC prognosis is unclear.

In this study, we compared the clinical and laboratory characteristics of patients with HCC with and without cirrhosis and found that high TG levels were independently related to $\mathrm{HCC}$ without cirrhosis. Furthermore, by multiple analyses, we identified high TG level to be an independent prognostic factor for better survival.

\section{Materials and methods}

\section{Patients}

A total of 3483 patients diagnosed with HCC in the medical records from February 2008 to December 2017 at the Beijing Ditan Hospital, Capital Medical University (Beijing, China) were retrospectively enrolled in the study.
The study was approved by the Committee of Ethics at Beijing Ditan Hospital, Capital Medical University. The inclusion criteria were as follows: (1) HCC diagnosed based on the pathology or serum $\alpha$-fetoprotein (AFP) level of $\geq 400 \mathrm{ng} / \mathrm{mL}$ in combination with imaging that showed typical appearances of HCC; (2) confirmed tumor staging of HCC, based on the Barcelona-Clinic Liver Cancer (BCLC) staging system; (3) had complete clinical data; (4) more than 1 year of follow-up. We excluded 955 patients with cholangiocarcinoma, metastatic liver cancer, or other types of cancer; those lost to follow-up, and those with short follow-up period (Fig. 1). We divided the final 2528 patients into subjects with and without cirrhosis according to the results of histopathology, clinical presentation, radiological studies, endoscopic examinations, and laboratory tests at the time of HCC diagnosis. We classified patients without cirrhosis if they meet the following criteria: 1) had liver biopsy-documented non-cirrhosis histology

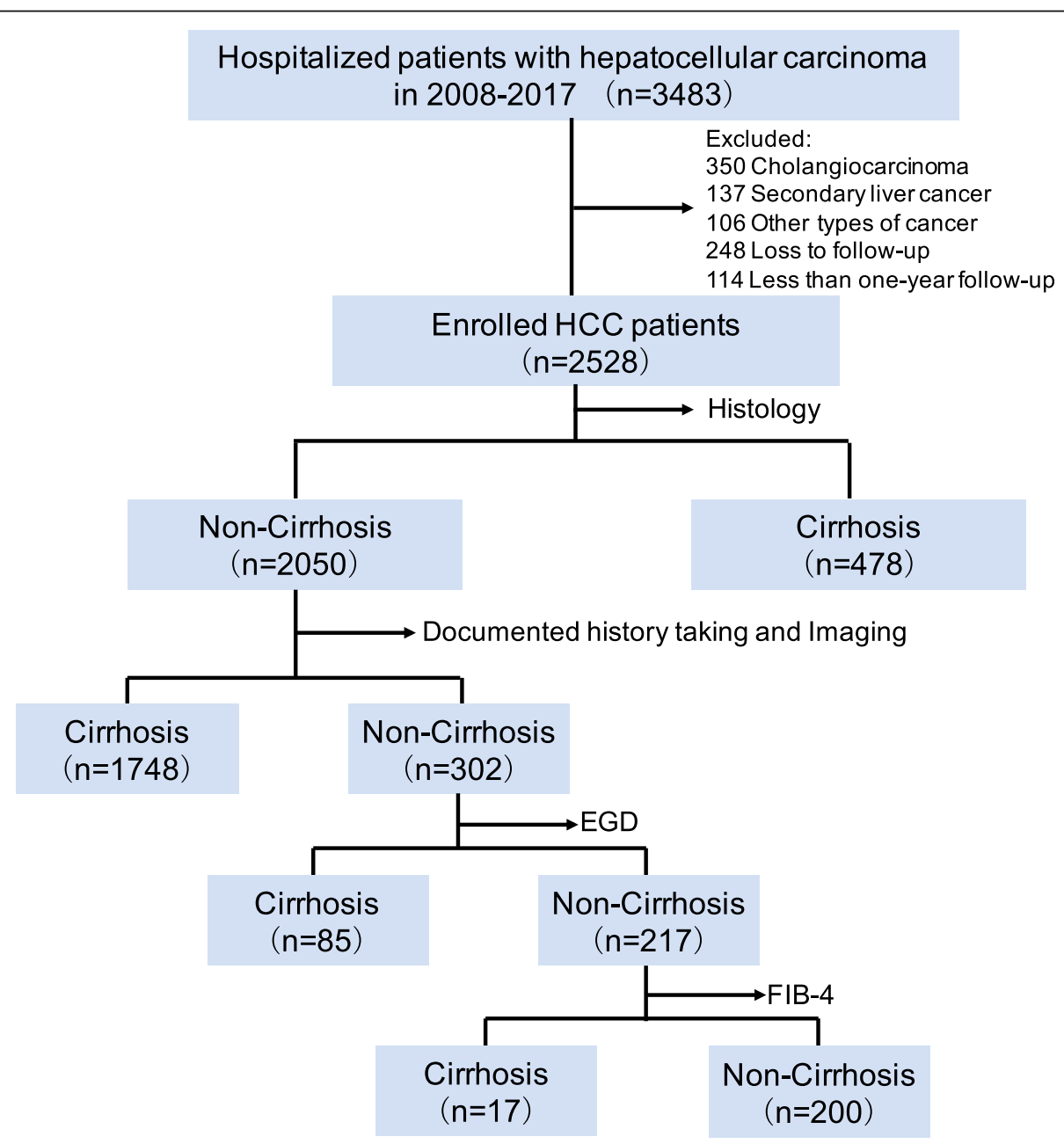

Fig. 1 Outline of the classification of HCC cohort study. Determination of cirrhosis status followed by laboratory tests, pathology, endoscopy and imaging studies. EGD, Esophagogastroduodenoscopy 
within 1 year before HCC diagnosis; 2) did not have clinical complications consisting of ascites, variceal bleeding, hepatorenal syndrome, and hepatic encephalopathy; 3) did not have cirrhosis related to morphologic changes and portal hypertension on imaging examination; 4) did not have esophageal and gastric varices on upper endoscopy; and 5) had FIB-4 value of $<1.45$. We calculated the FIB-4 score using laboratory results within 1 year before $\mathrm{HCC}$ diagnosis using the following formula: age (years) $\times$ aspartate aminotransferase $(\mathrm{U} / \mathrm{L}) /$ platelet $(\mathrm{PLT})\left(10^{9} / \mathrm{L}\right) \times$ alanine aminotransferase $(\mathrm{U} / \mathrm{L})^{1 / 2}$.

\section{Statistical analysis}

The statistical analyses were performed using SPSS version 21.0 software. Data were expressed as median and range for non-normal distribution and mean \pm standard deviation (SD) for normal distribution. Comparisons of patient characteristics were analyzed using Student's t-test for normal distribution and Mann-Whitney $U$ test for non-normal distribution. Categorical variables were assessed by Pearson $\mathrm{X}^{2}$ or Fisher's exact test. One-way ANOVA test followed by Tukey's multiple comparison test was performed for lipid profiles across the 3 groups (no cirrhosis, compensated cirrhosis, and decompensated cirrhosis). For all analyses, $P$ values of $<0.05$ were considered statistically significant.

The univariate and multivariate logistic regression analyses were performed to identify factors that were independently associated with patients with HCC without cirrhosis. We used Kaplan-Meier curves to estimate overall survival (OS) and progression-free survival (PFS) of different groups and compared survival curves using the log-rank test. The Cox proportional hazard regression analysis was used to determine the variables associated with the prognosis in patients with HCC without cirrhosis.

\section{Results}

\section{Baseline characteristics}

Between 2008 and 2017, we identified 2528 patients with HCC, of whom 2328 (92.1\%) had cirrhosis before or at the time of HCC diagnosis and 200 (7.9\%) did not have cirrhosis. Figure 1 shows the flowchart used to classify patients into the cirrhosis categories.

The demographic characteristics and clinical and biochemical characteristics of patients with and without cirrhosis are presented in Table 1. At the time of HCC diagnosis, patients without cirrhosis were 3.6 years younger than those with cirrhosis. Patients without cirrhosis had two times greater proportions of family history of HCC and history of hyperlipidemia than those without cirrhosis. Regarding the etiology, a greater proportion of patients with HCC without cirrhosis had NAFLD and idiopathic condition with no etiologic risk factor, and a lower proportion had alcoholic liver disease compared with patients with cirrhosis. In all patients with $\mathrm{HCC}, \mathrm{HBV}$ and $\mathrm{HCV}$ infections were both great risk factors. Patients with $\mathrm{HCC}$ in the absence of cirrhosis had a larger proportion of solitary tumor nodule (72\% vs $55.1 \%, p<0.0001)$ and a significantly lower prevalence of portal vein thrombosis (PVTT) at the time of HCC diagnosis $(7.0 \%$ vs $20.6 \%, p<0.0001)$ compared to those with underlying cirrhosis. The diameter of tumor showed no difference between HCC patients without cirrhosis (median, $3.5 \mathrm{~cm}$ ) and patients with cirrhosis (median, $3.1 \mathrm{~cm})(p=0.213)$. Patients with HCC in the absence of cirrhosis were more likely to have BCLC stage 0-A tumor (vs C or D) when compared with patients with cirrhosis. Moreover, HCC patients without cirrhosis received more resection treatment and less palliative care than patients with cirrhosis $(p=0.002)$. The median MELD scores were lower in patients with HCC without cirrhosis (median score, 3.4) than those with cirrhosis (median score, 5.39) $(p<0.0001)$. There were high levels of leukocyte counts, platelet counts, albumin, triglyceride, and prothrombin activity and low ratio of neutrophil to lymphocyte and low levels of aspartate aminotransferase (AST), total bilirubin (TBIL), and $\gamma$-glutamyl transferase (GGT) in patients with HCC without cirrhosis $(p<0.0001)$. There were no differences in other patient characteristics by cirrhosis status.

\section{Factors associated with HCC without cirrhosis}

Table 2 shows the results of the univariate and multivariate logistic regression analyses. Compared to patients with $\mathrm{HCC}$ with cirrhosis, patients with $\mathrm{HCC}$ without cirrhosis were statistically significantly more likely to be younger ( $\leq 50$ years), in Child A stage and have a family history of HCC and PVTT in the baseline. Patients with HCC without cirrhosis also frequently had higher platelet counts $\left(\geq 100 * 10^{9} / \mathrm{L}\right)$, higher TG level $(\geq 1.71 \mathrm{mmol} /$ $\mathrm{L})$, and more normal GGT level $(<60 \mathrm{U} / \mathrm{L})(P<0.05$ for all comparisons). Furthermore, we compared the lipid parameters in HCC patients with different status of cirrhosis and found the TG decreased in decompensated cirrhosis than non-cirrhosis and compensated cirrhosis $(\mathrm{P}<0.05$, Additional file 1: Table S1).

\section{Survival analysis}

The median overall survival time was 42 months for non-cirrhosis HCC patients and 25 months for HCC with cirrhosis cohort, respectively. There was a significantly longer OS in patients without cirrhosis compared to patients with cirrhosis (hazard ratio [HR], 0.67; 95\% CI, 0.57-0.80; $P<0.0001$; Fig. 2a); the 1-, 3-, and 5- year OS was 74.46, 55.23 and $40.61 \%$, respectively, in the non-cirrhosis group and 65.22, 38.30, and $23.93 \%$, respectively, in the cirrhosis group. The result 
Table 1 Demographic data and clinical characteristics of patients with hepatocellular carcinoma

\begin{tabular}{lll}
\hline & Non-cirrhosis $n=200(\%)$ & Cirrhosis $n$ \\
\hline $\begin{array}{l}\text { Age }(\text { mean } \pm \text { SD) } \\
\text { Gender (male) }\end{array}$ & $53.36 \pm 11.94$ & $56.96 \pm 10.34$ \\
$\begin{array}{l}\text { Family history of HCC } \\
\text { Yes }\end{array}$ & $164(82.0)$ & $1793(77.0)$ \\
$\quad$ No & $14(7.0)$ & $74(3.2)$ \\
Smoking & $186(93.0)$ & $2254(96.8)$ \\
$\quad$ & & \\
Smoker & $91(45.5)$ & $1396(60.0)$ \\
Non-smoker & $109(54.5)$ & $894(38.4)$ \\
Alcohol & & $1434(61.6)$ \\
$\quad$ alcohol & $79(39.5)$ & \\
No alcohol & $121(60.5)$ & $506(21.7)$ \\
Diabetes & & $1822(78.3)$ \\
$\quad$ Yes & $21(10.5)$ &
\end{tabular}

Hypertension

Yes

No

Hyperlipidemia

Yes

No

Coronary artery disease

Yes

No

Etiology

HBV

$\mathrm{HCV}$

Alcohol abuse

NAFLD

idopathic

$\mathrm{HBeAg}$ at baseline

Negative

Positive

Missing data

HBV-DNA at baseline

$$
\begin{aligned}
& \operatorname{Low}(<500 \mathrm{IU} / \mathrm{ml}) \\
& \text { High(> } 500 \mathrm{IU} / \mathrm{ml}) \\
& \text { Missing data }
\end{aligned}
$$

Antiviral therapy

Yes

No

Missing data

Tumor diameter $(\mathrm{cm})$

Tumor multiplicity

solitary
50 (25)

150 (75)

25 (12.5)

$175(87.5)$

$5(2.5)$

195 (97.5)

$153(76.5)$

23 (11.5)

$10(5.0)$

$4(2.0)$

$10(5.0)$

$98(54.1)$

45 (24.9)

$38(21.0)$

71 (41.3)

63 (36.6)

38 (22.1)

146 (79.3)

29 (15.8)

9 (4.9)

$3.5(2.3,6.2)$

136 (72)
$P$ values

0.106

0.005

0.759

$<0.0001$

0.81

$600(25.8)$

$1728(74.2)$

0.002

154 (6.6)

$2174(93.4)$

61 (2.6)

2267 (97.4)

0.003

$1823(78.3)$

177 (7.6)

248 (10.7)

25 (1.1)

55 (2.4)

0.005

$1289(60.6)$

572 (26.9)

265 (12.5)

0.024

791 (39.8)

$894(45.0)$

301 (15.2)

0.972

$1686(79.0)$

348 (16.3)

$99(4.6)$

$3.1(2.0,5.7)$

0.213

$<0.0001$

$1259(55.1)$ 
Table 1 Demographic data and clinical characteristics of patients with hepatocellular carcinoma (Continued)

\begin{tabular}{|c|c|c|c|}
\hline & Non-cirrhosis $n=200(\%)$ & Cirrhosis $n=2328(\%)$ & $P$ values \\
\hline multiple & $53(28)$ & $1024(44.9)$ & \\
\hline PVTT at baseline & & & $<0.0001$ \\
\hline Yes & $14(7.0)$ & $479(20.6)$ & \\
\hline No & $186(93.0)$ & $1849(79.4)$ & \\
\hline AFP $(n g / m l)$ & & & 0.754 \\
\hline AFP $<400$ & $148(74.0)$ & $1746(74.9)$ & \\
\hline$A F P \geq 400$ & $52(26.0)$ & $634(25.1)$ & \\
\hline BCLC staging & & & $<0.0001$ \\
\hline $0-A$ & $106(53.0)$ & $835(37.3)$ & \\
\hline B & $73(36.5)$ & $734(31.9)$ & \\
\hline C & $18(9.0)$ & $433(17.8)$ & \\
\hline D & $3(1.5)$ & $326(13.0)$ & \\
\hline Treatment for HCC & & & 0.002 \\
\hline Resection & $30(15.0)$ & $185(7.9)$ & \\
\hline Minimally invasive & $132(66.0)$ & $1578(67.8)$ & \\
\hline Palliative & $38(19.0)$ & $565(24.3)$ & \\
\hline MELD scores & $3.4(1.29,5.30)$ & $5.39(2.36,8.48)$ & $<0.0001$ \\
\hline Leukocyte counts $\left(10^{9} / \mathrm{L}\right)$ & $4.43(5.71,7.25)$ & $3.05(4.32,5.90)$ & $<0.0001$ \\
\hline NLR & $2.19(1.55,3.68)$ & $2.44(1.62,3.97)$ & 0.019 \\
\hline Platelets $\left(10^{9} / \mathrm{L}\right)$ & $151.8(112.5197 .08)$ & $87.25(57.23,136.5)$ & $<0.0001$ \\
\hline ALT $(U / L)$ & $32.1(22.72,56.7)$ & $33.2(22.3,54.2)$ & 0.9 \\
\hline AST (U/L) & $31.15(23.8,54.03)$ & $42.4(28.83,72.13)$ & $<0.0001$ \\
\hline Totall Bilirubin (umol/L) & $13.4(9.7,16.98)$ & $19.85(13.2,32.48)$ & $<0.0001$ \\
\hline$\gamma-G G T(U / L)$ & $44.9(25.9,94.33)$ & $59.35(33.22,123.8)$ & $<0.0001$ \\
\hline Albumin (g/L) & $40.09 \pm 4.80$ & $35.014 \pm 6.34$ & $<0.0001$ \\
\hline Triglyceride (mmol/L) & $1.009 \pm 0.49$ & $0.87 \pm 0.45$ & $<0.0001$ \\
\hline Prothrombin activity (\%) & $88.77 \pm 14.51$ & $75.06 \pm 18.00$ & $<0.0001$ \\
\hline Child Staging & & & $<0.0001$ \\
\hline A & $177(88.5)$ & $1125(48.3)$ & \\
\hline B & $20(10.0)$ & $877(37.7)$ & \\
\hline C & $3(1.5)$ & $326(14.0)$ & \\
\hline
\end{tabular}

Abbreviations: SD, standard deviation; PVTT, portal vein tumor thrombus; AFP, alpha-fetoprotein; NLR, Neutrophil-lymphocyte ratio; ALT, alanine aminotransferase; AST, aspartate aminotransferase; $\gamma$-GGT $\gamma$-glutamyl transferase

of progress-free survival rate (PFS) was consistent with the OS (hazard ratio [HR], 0.66; 95\% CI, 0.57-0.78; $P<$ 0.0001; Figure 2b). In exploratory subgroup analysis, the group without cirrhosis had an PFS benefit than those with cirrhosis in both subgroups of patients with TG level $\geq 1.71 \mathrm{mmol} / \mathrm{L}$ and $<1.71 \mathrm{mmol} / \mathrm{L}$ (Fig. $3 \mathrm{~b}$ and d). Similarly, patients without cirrhosis had significantly better OS than those without cirrhosis in patients with TG $<1.71 \mathrm{mmol} / \mathrm{L}$ (Fig. 3a), but the benefit of OS was not remarkable in TG $\geq 1.71 \mathrm{mmol} / \mathrm{L}$ group $(P=$ 0.053, Fig. 3c).

\section{Risk factors of death in patients with HCC without cirrhosis}

Table 3 displays the results of univariate and multivariate Cox proportional hazard regression analysis. By univariate analysis, alcohol abuse, presence of PVTT at the time of HCC diagnosis, high TBIL level, high GGT level, AFP level of $\geq 400 \mathrm{ng} / \mathrm{mL}$, tumor size of $\geq 5 \mathrm{~cm}$, and tumor number of $\geq 2$ were found to be significant risk factors, and a high albumin level and antiviral therapy were the protective factors for the incidence of death in patients without cirrhosis. By the multivariate analysis, we found 
Table 2 Factors associated with hepatocellular carcinoma in the absence of cirrhosis

\begin{tabular}{|c|c|c|c|c|c|c|}
\hline \multirow[t]{2}{*}{ Variables } & \multicolumn{3}{|c|}{ Univariate analysis } & \multicolumn{3}{|c|}{ Multivariate analysis } \\
\hline & $\mathrm{OR}$ & $95 \% \mathrm{Cl}$ & $P$ values & $\mathrm{OR}$ & $95 \% \mathrm{Cl}$ & $P$ values \\
\hline Age $>50$ yrs & 0.46 & $0.34-0.62$ & $<0.0001$ & 0.42 & $0.25-0.70$ & $<0.0001$ \\
\hline Gender (male) & 1.36 & $0.94-1.98$ & 0.11 & & & \\
\hline Family history of HCC & 2.29 & $1.27-4.14$ & 0.006 & 2.91 & $1.07-7.92$ & 0.037 \\
\hline Diabetes & 0.59 & $0.39-0.89$ & 0.012 & & & \\
\hline Hyperlipidemia & 2.02 & $1.29-3.16$ & 0.002 & & & \\
\hline \multicolumn{7}{|l|}{ Etiology } \\
\hline HBV & 0.79 & $0.54-1.13$ & 0.19 & & & \\
\hline $\mathrm{HCV}$ & 1.43 & $0.92-2.22$ & 0.12 & & & \\
\hline Alcohol abuse & 0.44 & $0.23-0.85$ & 0.014 & & & \\
\hline NAFLD & 6.77 & $2.0-23.32$ & 0.002 & & & \\
\hline HBV-DNA $\geq 500 \mathrm{IU} / \mathrm{ml}$ & 0.79 & $0.55-1.11$ & 0.18 & & & \\
\hline Antiviral therapy & 1.04 & $0.69-1.57$ & 0.86 & & & \\
\hline PVTT at baseline & 0.29 & $0.17-0.51$ & $<0.0001$ & & & \\
\hline Leukocyte counts $\geq 4^{*} 10^{9} / \mathrm{L}$ & 2.31 & $1.79-2.98$ & $<0.0001$ & & & \\
\hline $\mathrm{PLT} \geq 100^{*} 10^{9} / \mathrm{L}$ & 6.23 & $4.31-9.02$ & $<0.0001$ & 6.67 & $3.32-13.40$ & $<0.0001$ \\
\hline $\mathrm{AST} \geq 40(\mathrm{U} / \mathrm{L})$ & 0.52 & $0.39-0.70$ & $<0.0001$ & & & \\
\hline $\mathrm{\gamma}-\mathrm{GGT} \geq 60(\mathrm{U} / \mathrm{L})$ & 0.57 & $0.42-0.77$ & $<0.0001$ & 0.46 & $0.27-0.78$ & 0.004 \\
\hline $\mathrm{TG} \geq 1.71 \mathrm{mmol} / \mathrm{L}$ & 2.46 & $1.50-4.02$ & $<0.0001$ & 2.66 & $1.18-6.01$ & 0.019 \\
\hline PTA $<70 \%$ & 0.18 & $0.11-0.29$ & $<0.0001$ & & & \\
\hline AFP $\geq 400 \mathrm{ng} / \mathrm{ml}$ & 1.05 & $0.76-1.47$ & 0.75 & & & \\
\hline Child staging & & & $<0.0001$ & & & \\
\hline A & 17.1 & $5.43-53.88$ & $<0.0001$ & 5.67 & $1.34-24.02$ & 0.019 \\
\hline B & 2.48 & $0.73-8.40$ & 0.15 & & & \\
\hline \multicolumn{7}{|l|}{ C (Reference) } \\
\hline BCLC staging & & & $<0.0001$ & & & \\
\hline $0-A$ & 13.8 & $4.35-43.77$ & $<0.0001$ & & & \\
\hline B & 10.81 & $3.38-34.54$ & $<0.0001$ & & & \\
\hline C & 4.52 & $1.32-15.47$ & 0.016 & & & \\
\hline D (Reference) & & & & & & \\
\hline
\end{tabular}

A

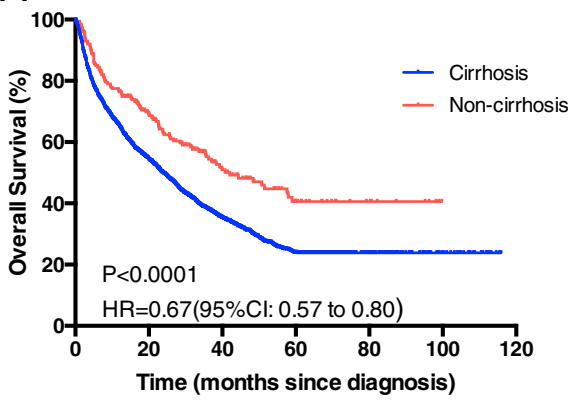

B

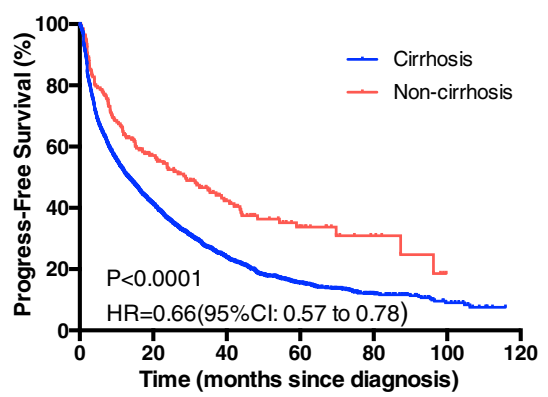

Fig. 2 Overall survival (a) and Progress-free survival (b) in HCC patients with and without cirrhosis 
A

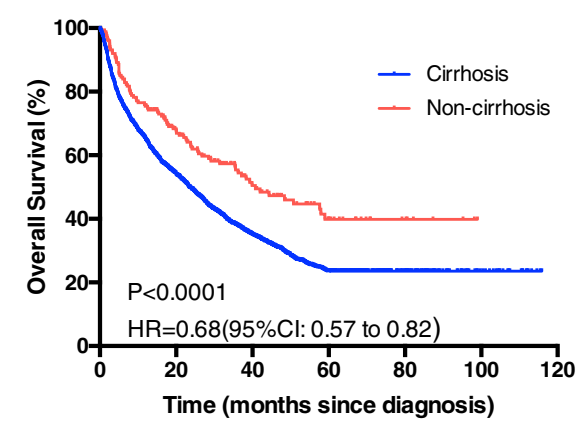

C

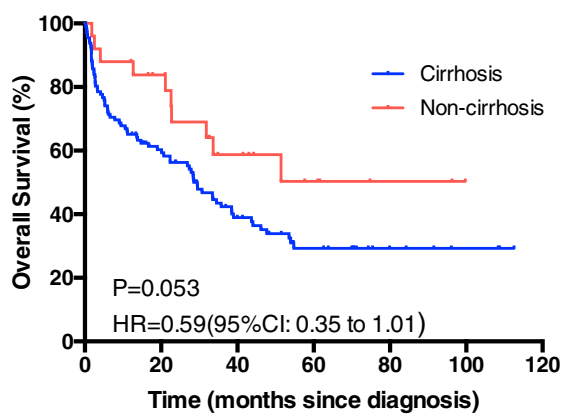

B

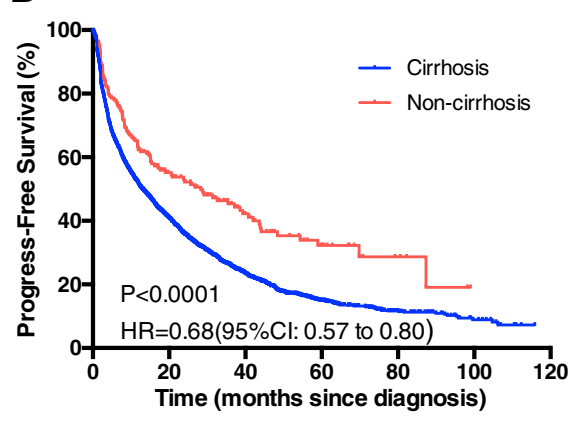

D

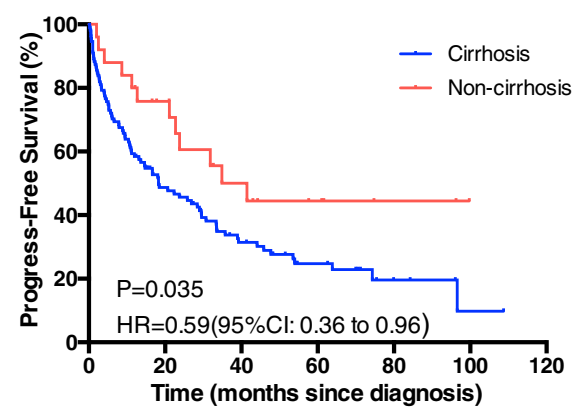

Fig. 3 Overall survival (OS) and Progress-free survival (PFS) in different triglyceride subgroups of HCC patients with and without cirrhosis. a-b The OS (a) and PFS (b) in triglyceride level $<1.7 \mathrm{mmol} / \mathrm{L}$ subgroup. $\mathbf{c}-\mathbf{d}$ The OS (c) and PFS (d) in triglyceride level $\geq 1.7 \mathrm{mmol} / \mathrm{L}$ subgroup

that PVTT at the time of HCC diagnosis (adjusted HR, 3.40; 95\% CI, 1.73-6.67), GGT (HR, 1.006; 95\% CI, 1.004-1.009), and tumor size of $>5 \mathrm{~cm}$ (HR, 2.79; 95\% $\mathrm{CI}, 1.72-4.50)$ remained the independent risk factors associated with a high risk of mortality. Furthermore, antiviral therapy ( $\mathrm{HR}, 0.53 ; 95 \% \mathrm{CI}, 0.32-0.89)$ and high serum TG levels (HR, 0.51; 95\% CI, 0.29-0.89) were the independent protective factors for the survival of patients with HCC without cirrhosis.

A further analysis was performed to evaluate the influence of each risk factor on the OS and PFS of patients with $\mathrm{HCC}$ without cirrhosis. We set $0.81 \mathrm{mmol} / \mathrm{L}$ as the triglyceride cut-off value which was the largest one for the Youden index. In the Kaplan-Meier analysis presented in Fig. 4, patients with HCC without cirrhosis with TG level $<0.81 \mathrm{mmol} / \mathrm{L}$ had worse OS and PFS compared to those with TG level of $\geq 0.81 \mathrm{mmol} / \mathrm{L}(P<0.0001$; Fig. $4 \mathrm{a}-\mathrm{b})$. We also examined the predictive value of serum TG level at different BCLC stages (Fig. 4c-f). For patients in stages 0 -A and patients in stages $\mathrm{B}, \mathrm{C}$ and $\mathrm{D}$, the OS of patients with TG level of $<0.81 \mathrm{mmol} / \mathrm{L}$ were significantly lower than those with TG level of $\geq 0.81 \mathrm{mmol} / \mathrm{L}$ throughout the follow-up period (Fig. 4c-f).

Combining the triglycerides level, cirrhosis status and age which are related to the serum triglycerides, it was possible to delineate 8 risk categories, with a 5.80-fold (95\% CI 3.19-10.52) increased probability of death in patients with HCC when the highest category (HCC patients with cirrhosis aged above 50 years and with triglycerides $<0.81 \mathrm{mmol} / \mathrm{L}$ ) was compared with the lowest category (HCC patients without cirrhosis aged < 50 years and with triglycerides $\geq 0.81 \mathrm{mmol} / \mathrm{L}$ ) (Table 4 ).

\section{Discussion}

Previous studies suggested that dyslipidemia was a newly identified risk factor in the survival of several cancers such as colorectal, breast, and prostate cancers $[15,16,18]$. Although one study demonstrated that preoperative HDL level was a predictor of $\mathrm{HCC}$ recurrence after liver resections [22]; recently, there were few studies that addressed the association between lipid profiles and the outcome of liver cancer. In our study, we found that a decreased TG level in the baseline was an independent risk factor of OS in patients with $\mathrm{HCC}$ without cirrhosis. A reduced serum TG level was related to worse OS and PFS by the optimal cutoff values using the ROC analysis.

Hypertriglyceridemia was previously considered to be closely related to a higher risk of cardiovascular disease [23]. However, some epidemiological researches have addressed the interrelation between serum TG and cancer risk lately. A high TG level has been linked to increased esophageal and colon cancer risks in a large-scale European cohort [24]. Other studies revealed a high TG level was inversely associated with prostate and breast cancer $[25,26]$. 
Table 3 Factors associated with overall survival of patients with HCC in the absence of cirrhosis

\begin{tabular}{|c|c|c|c|c|c|c|}
\hline \multirow[t]{2}{*}{ Variables } & \multicolumn{3}{|c|}{ Univariate analysis } & \multicolumn{3}{|c|}{ Multivariate analysis } \\
\hline & $\mathrm{HR}$ & $95 \% \mathrm{Cl}$ & $P$ values & $\mathrm{HR}$ & $95 \% \mathrm{Cl}$ & $P$ values \\
\hline Age $>50$ yrs & 1.01 & $0.99-1.03$ & 0.283 & & & \\
\hline Gender (male) & 0.85 & $0.51-1.40$ & 0.517 & & & \\
\hline Family history of HCC & 1.16 & $0.51-2.67$ & 0.72 & & & \\
\hline Alcohol abuse & 1.76 & $1.18-2.63$ & 0.006 & & & \\
\hline Diabetes & 1.41 & $0.83-2.38$ & 0.202 & & & \\
\hline Hypertension & 0.85 & $0.53-1.36$ & 0.507 & & & \\
\hline Hyperlipidemia & 0.98 & $0.54-1.80$ & 0.949 & & & \\
\hline \multicolumn{7}{|l|}{ Etiology } \\
\hline HBV & 0.62 & $0.39-0.97$ & 0.037 & & & \\
\hline $\mathrm{HCV}$ & 1.8 & $1.08-3.02$ & 0.025 & & & \\
\hline Alcohol abuse & 1.54 & $0.71-3.32$ & 0.272 & & & \\
\hline NAFLD & 2.07 & $0.65-6.54$ & 0.216 & & & \\
\hline HBV-DNA $\geq 500 \mathrm{IU} / \mathrm{ml}$ & 1.36 & $0.88-2.10$ & 0.164 & & & \\
\hline Antiviral therapy & 0.52 & $0.32-0.86$ & 0.011 & 0.53 & $0.32-0.89$ & 0.015 \\
\hline PVTT at baseline & 6.17 & $3.32-11.46$ & $<0.0001$ & 3.4 & $1.73-6.67$ & $<0.0001$ \\
\hline Leukocyte counts & 1.05 & $0.96-1.14$ & 0.332 & & & \\
\hline NLR & 0.99 & $0.95-1.03$ & 0.675 & & & \\
\hline Platelets $\left(10^{9} / \mathrm{L}\right)$ & 1.002 & $0.999-1.005$ & 0.193 & & & \\
\hline AST (U/L) & 1.002 & $0.998-1.006$ & 0.403 & & & \\
\hline Totall Bilirubin (umol/L) & 1.005 & $1.002-1.008$ & 0.001 & & & \\
\hline Albumin (g/L) & 0.939 & $0.902-0.978$ & 0.002 & & & \\
\hline$\gamma-G G T(U / L)$ & 1.006 & $1.004-1.008$ & $<0.0001$ & 1.006 & $1.004-1.009$ & $<0.0001$ \\
\hline Triglyceride (mmol/L) & 0.7 & $0.44-1.11$ & 0.128 & 0.51 & $0.29-0.89$ & 0.017 \\
\hline PTA (\%) & 0.986 & $0.973-0.999$ & 0.032 & & & \\
\hline AFP $\geq 400 \mathrm{ng} / \mathrm{ml}$ & 2.235 & $1.469-3.40$ & $<0.0001$ & & & \\
\hline Tumor size $>5 \mathrm{~cm}$ & 3.99 & $2.65-6.02$ & $<0.0001$ & 2.79 & $1.72-4.50$ & $<0.0001$ \\
\hline Tumor numbers $\geq 2$ & 2.3 & $1.53-3.46$ & $<0.0001$ & & & \\
\hline $\mathrm{CRP}(\mathrm{mg} / \mathrm{L})$ & 1.008 & $1.003-1.013$ & 0.002 & & & \\
\hline
\end{tabular}

Abbreviations: CRP C-reactive protein

It is inconsistent with the relationship between serum TG concentration and HCC risk. A large prospective cohort study with a long follow-up period showed that elevated TG level contributed to an increased risk of primary liver cancer in patients who had more than $50 \%$ history of alcohol liver disease [19]. Another study indicated that the TG level was strongly associated with reduced risk of HBV-related HCC [27]. The association between TG and HCC remains unclear. To our knowledge, this is the first time to report a negative association between TG levels and HCC death. In this study, we have shown that patients with $\mathrm{HCC}$ in the absence of cirrhosis with TG level < $0.81 \mathrm{mmol} / \mathrm{L}$ had a worse OS and PFS than patients with TG level $\geq 0.81 \mathrm{mmol} / \mathrm{L}(P<0.0001)$. The normal cutoff values for TG may be interpreted by the fact that the serum TG level decreased by $20-30 \%$ in patients with HCC compared to healthy participants $[28,29]$. In contrast, the pro-inflammatory cytokines like interleukin-6 (IL-6), IL-1, and tumor necrosis factor alpha (TNF- $\alpha$ ) secreted by tumor cells may inhibit TG synthesis [30, 31]. On the contrary, increased adipose TG lipase and hormone-sensitive lipase activity in cancer may promote the complete hydrolysis of TG molecule to free fatty acids (FFA), which can provide substrates for the proliferation of tumor cells [32, 33]. Suppressed synthesis and excessive hydrolysis play an important role in dyslipidemia. Decreased levels of TG have been connected with increased circulating levels of IL-6, TNF- $\alpha$ which are thought to induced the tumor cell proliferation and inhibit apoptosis [34]. In addition, the high death rate linked with decreased TG concentration may be a consequence of cancer-associated cachexia. However, the exact mechanisms require further investigation. 


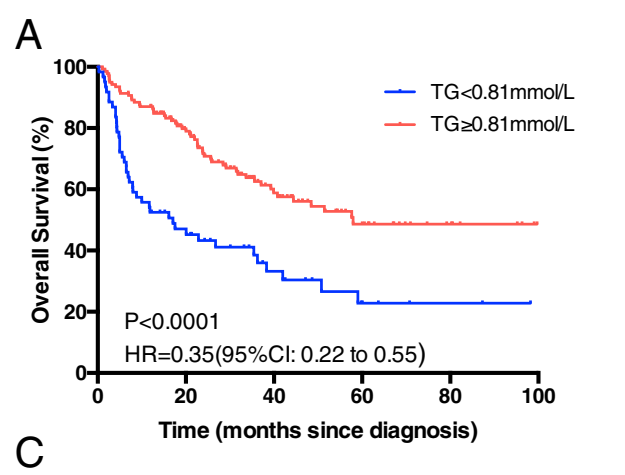

B
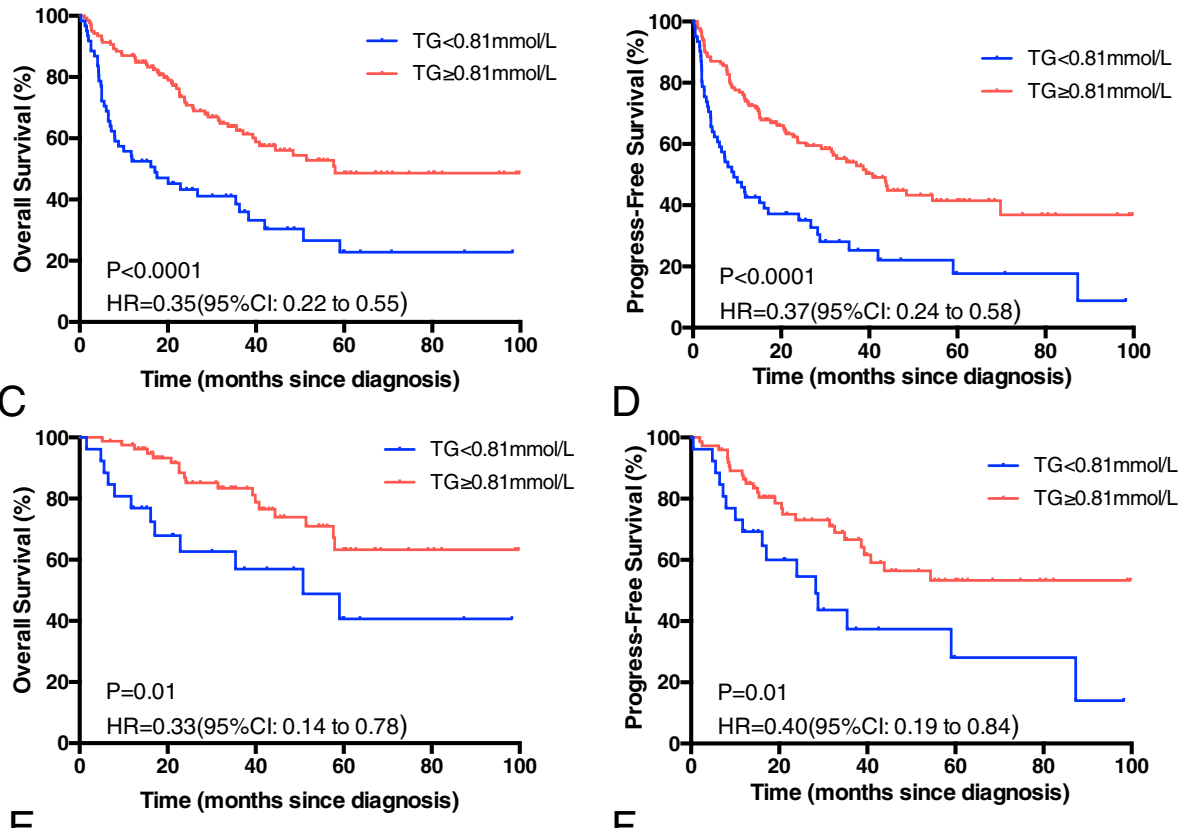

$\mathrm{E}$

$\mathrm{F}$
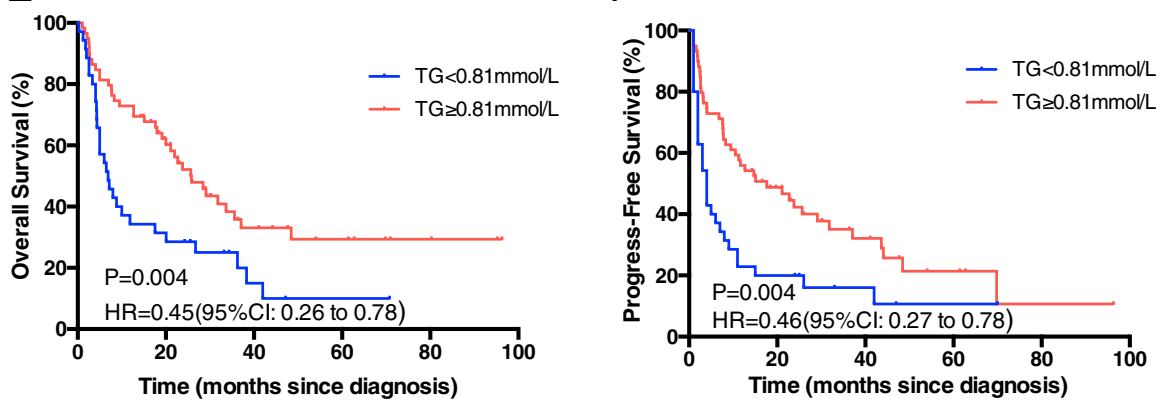

Fig. 4 Kaplan-Meier curve analysis showing overall survival (OS) and progress-free survival (PFS) of different triglyceride level in HCC patients without cirrhosis. a-b The OS (a) and PFS (b) for all HCC patients without cirrhosis. c-d The OS (c) and PFS (d) for HCC patients without cirrhosis with BCLC stage 0 and A. e-f The OS (e) and PFS (f) for HCC patients without cirrhosis with BCLC stage B, C and D

We also found that there were some differences in lipid, lipoproteins, and apolipoproteins in patients with HCC with and without cirrhosis. Patients with HCC without cirrhosis had increased triglyceride, cholesterol, HDL-C, LDL-C, and ApoB levels compared to patients with cirrhosis. More profoundly, the decrease of lipid components was more obvious in patients with HCC with decompensated cirrhosis than those with compensated cirrhosis. These results were consistent with a previous study that lowering of lipoprotein levels was significantly linked to the increasing severity of liver disease [35, 36]. Besides, using multiple logistic regression analysis, portal vein thrombosis and tumor size $>5 \mathrm{~cm}$ at $\mathrm{HCC}$ diagnosis were associated with poor prognosis, consistent with previous studies in several cancers [37]. We also found that patients with HCC without cirrhosis had elevated serum TG levels $(\mathrm{OR}=2.66)$. The liver plays a key role in the synthesis and metabolism of lipids and lipoprotein. Hepatic FA derive form endogenous lipogenesis and the FFA plasma pool are processed to triacylglycerols and stored or rapidly

Table 4 Risk of overall survival according to cirrhosis status, age, triglyceride level in patients with hepatocellular carcinoma

\begin{tabular}{|c|c|c|c|c|}
\hline & \multicolumn{2}{|l|}{ Non-cirrhosis } & \multicolumn{2}{|l|}{ Cirrhosis } \\
\hline & Age $<50$ yrs & Age $\geq 50$ yrs & Age $<50$ yrs & Age $\geq 50$ yrs \\
\hline $\mathrm{TG}<0.81 \mathrm{mmol} / \mathrm{L}$ & $7.48(3.58-15.61) P<0.0001$ & $3.87(1.87-8.03) P<0.0001$ & $5.65(3.07-10.39) P<0.0001$ & $5.80(3.19-10.52) P<0.0001$ \\
\hline $\mathrm{TG} \geq 0.81 \mathrm{mmol} / \mathrm{L}$ & $1^{*}$ & $2.93(1.51-5.68) P=0.001$ & $3.55(1.93-6.54) P<0.0001$ & $3.76(2.07-6.82) P<0.0001$ \\
\hline
\end{tabular}

Results are expressed as hazard ratios (95\% confidence intervals). ${ }^{*}$ Reference category 
metabolized in hepatocyte [38]. Liver cirrhosis due to long-term virus infections causes greater hepatocyte necrosis and influences the lipid concentration. Our finding may provide new evidence that patients with cirrhosis developed more serious liver injury and dyslipidemia. The better liver function reflected by a higher TG level may explain the better survival in patients with $\mathrm{HCC}$ without cirrhosis than those with cirrhosis.

Our study had some limitations. First, the variables analyzed in our study did not have a treatment strategy, which was an important factor affecting the prognosis. The influence of different clinical interventions on the lipid profiles need to be clarified in future studies. Second, HBV infection is the main risk factor for the incidence of HCC in China. The racial differences in TG levels should be considered, and the cutoff value needs to be redefined when generalizing the results to people with different risk factors for HCC. Finally, the patients in our study were mostly men, and the prognostic effect of TG in women may be limited.

In conclusion, this study revealed that TG levels at the time of HCC diagnosis may be considered as independent prognostic factor for liver cancer. These results indicate that some appropriate treatments may be applied to adjust lipids to normal or high levels.

\section{Additional file}

Additional file 1: Table S1. Changes of lipid parameters in HCC patients without cirrhosis, compensated cirrhosis and decompensated cirrhosis (DOCX $14 \mathrm{~kb}$ )

\section{Acknowledgements}

Not applicable.

\section{Availability of data and material}

The datasets used or analyzed during the current study are available from the corresponding author on reasonable request.

\section{Funding}

This work was supported by the Fund of Special research of TCM in Capital City (17ZY02); the Fund for Beijing Science \& Technology Development of TCM (No. JJ2016-14); and Application of Clinical Features of Capital City of Science and Technology Commission (No. Z171100001017082).

\section{Authors' contributions}

ZYY designed the study; XLL collected and analyzed the data and wrote the manuscript; MGL, XHW and ZBD provided patients data; YYJ and XBW were responsible for the interpretation of data and revision. $Z Y Y$ approved for final revision and approval.

\section{Ethics approval and consent to participate}

The study was approved by the ethics committee of Beijing Ditan Hospital, Capital Medical University.

\section{Consent for publication}

Written informed consent was obtained from each patient. Information that could identify individual participants during or after data collection was not accessible.

\section{Competing interests}

The authors declare that they have no competing interests. We declare that we do not have any commercial or associative interest that represents a conflict of interest in connection with the work submitted.

\section{Publisher's Note}

Springer Nature remains neutral with regard to jurisdictional claims in published maps and institutional affiliations.

Received: 12 August 2018 Accepted: 23 October 2018

Published online: 06 November 2018

\section{References}

1. Njei B, Rotman $Y$, Ditah I, et al. Emerging trends in hepatocellular carcinoma incidence and mortality[J]. Hepatology. 2015;61(1):191-9.

2. Torre LA, Bray F, Siegel RL, et al. Global cancer statistics, 2012[J]. CA Cancer J Clin. 2015;65(2):87-108.

3. Yang JD, Kim WR, Coelho $R$, et al. Cirrhosis is present in most patients with hepatitis B and hepatocellular carcinoma[J]. Clin Gastroenterol Hepatol. 2011;9(1):64-70.

4. Fattovich G, Bortolotti F, Donato F. Natural history of chronic hepatitis B: special emphasis on disease progression and prognostic factors[J]. J Hepatol. 2008;48(2):335-52.

5. Trevisani F, Frigerio M, Santi V, et al. Hepatocellular carcinoma in noncirrhotic liver: a reappraisal[J]. Dig Liver Dis. 2010;42(5):341-7.

6. Wang X, Wang N, Cheung F, et al. Chinese medicines for prevention and treatment of human hepatocellular carcinoma: current progress on pharmacological actions and mechanisms[J]. Journal of integrative medicine. 2015;13(3):142-64.

7. Li M, Tang Y, Wu L, et al. Hepatocyte-specific HNF4a/miR-122 pathway contributes to the iron-overload mediated hepatic inflammation[J]. Blood. 2017;130(8):1041-51

8. Uccello M, Malaguarnera G, Pelligra EM, et al. Lipoprotein (a) as a potential marker of residual liver function in hepatocellular carcinoma[J]. Indian journal of medical and paediatric oncology: official journal of Indian Society of Medical \& Paediatric. Oncology. 2011:32(2):71.

9. Lee S, Mardinoglu A, Zhang C, et al. Dysregulated signaling hubs of liver lipid metabolism reveal hepatocellular carcinoma pathogenesis[J]. Nucleic Acids Res. 2016;44(12):5529-39.

10. Morsy KH, Ghaliony MAA, Kobeisy MA. Lipid profile among cirrhotic patients with and without hepatocellular carcinoma in upper Egypt[J]. Journal of the Arab Society for Medical Research. 2012;7(1):33-7.

11. Ooi K, Shiraki $K$, Sakurai $Y$, et al. Clinical significance of abnormal lipoprotein patterns in liver diseases[J]. Int J Mol Med. 2005;15(4):655-60.

12. Chen $S$, Yin $P$, Z Zhao $X$, et al. Serum lipid profiling of patients with chronic hepatitis B, cirrhosis, and hepatocellular carcinoma by ultra fast LC/IT-TOF MS[J]. Electrophoresis. 2013;34(19):2848-56.

13. Lin $X, L u L$, Liu L, et al. Blood lipids profile and lung cancer risk in a metaanalysis of prospective cohort studies[J]. Journal of clinical lipidology. 2017; 11(4):1073-81.

14. Vílchez JA, Martínez-Ruiz A, Sancho-Rodríguez N, et al. The real role of prediagnostic high-density lipoprotein cholesterol and the cancer risk: a concise review[J]. Eur J Clin Investig. 2014;44(1):103-14.

15. Goulart A, Varejão A, Nogueira F, et al. The influence of metabolic syndrome in the outcomes of colorectal cancer patients[J]. Diabetes \& Metabolic Syndrome: Clinical Research \& Reviews. 2017;11:S867-71.

16. Li X, Tang H, Wang J, et al. The effect of preoperative serum triglycerides and high-density lipoprotein-cholesterol levels on the prognosis of breast cancer[J]. Breast. 2017;32:1-6.

17. Chandler PD, Song Y, Lin J, et al. Lipid biomarkers and long-term risk of cancer in the Women's health study-3[J]. Am J Clin Nutr. 2016; 103(6):1397-407.

18. Bull CJ, Bonilla C, Holly JMP, et al. Blood lipids and prostate cancer: a Mendelian randomization analysis[J]. Cancer medicine. 2016;5(6):1125-36.

19. Nderitu P, Bosco C, Garmo H, et al. The association between individual metabolic syndrome components, primary liver cancer and cirrhosis: a study in the Swedish AMORIS cohort[J]. Int J Cancer. 2017;141:1148-60.

20. Khattab MA, Eslam M, Mousa Yl, et al. Association between metabolic abnormalities and hepatitis C-related hepatocellular carcinoma[J]. Ann Hepatol. 2012;11(4):487-94. 
21. Zhao J, Zhao Y, Wang H, et al. Association between metabolic abnormalities and HBV related hepatocelluar carcinoma in Chinese: a cross-sectional study[]]. Nutr J. 2011;10(1):49.

22. Tian $\mathrm{L}, \mathrm{Yu} \mathrm{Q}, \mathrm{Gao} X \mathrm{XH}$, et al. A new use for an old index: preoperative highdensity lipoprotein predicts recurrence in patients with hepatocellular carcinoma after curative resections[J]. Lipids Health Dis. 2017;16(1):123.

23. Tenenbaum A, Klempfner R, Fisman EZ. Hypertriglyceridemia: a too long unfairly neglected major cardiovascular risk factor[]]. Cardiovasc Diabetol. 2014;13(1):159

24. Wulaningsih W, Garmo H, Holmberg L, et al. Serum lipids and the risk of gastrointestinal malignancies in the Swedish AMORIS study[J]. Journal of cancer epidemiology. 2012;2012. https://doi.org/10.1155/2012/792034.

25. Van Hemelrijck $M$, Garmo H, Holmberg L, et al. Prostate cancer risk in the Swedish AMORIS study[J]. Cancer. 2011;117(10):2086-95.

26. Vatten $L$, Foss OP. Total serum cholesterol and triglycerides and risk of breast cancer: a prospective study of 24,329 Norwegian women[J]. Cancer Res. 1990;50(8):2341-6.

27. Chen $\mathrm{CL}$, Yang HI, Yang WS, et al. Metabolic factors and risk of hepatocellular carcinoma by chronic hepatitis B/C infection: a follow-up study in Taiwan[J]. Gastroenterology. 2008;135(1):111-21.

28. Motta M, Giugno I, Ruello P, et al. Lipoprotein (a) behaviour in patients with hepatocellular carcinoma[J]. Minerva Med. 2001:92(5):301-5.

29. Jiang J, Nilsson-Ehle P. Xu N. influence of liver cancer on lipid and lipoprotein metabolism[J]. Lipids Health Dis. 2006;5(1):4.

30. Chen X, Xun K, Chen L, et al. TNF- $a$, a potent lipid metabolism regulator[J]. Cell Biochem Funct. 2009:27(7):407-16.

31. Park EJ, Lee JH, Yu GY, et al. Dietary and genetic obesity promote liver inflammation and tumorigenesis by enhancing IL-6 and TNF expression[J]. Cell. 2010;140(2):197-208

32. Currie E, Schulze A, Zechner R, et al. Cellular fatty acid metabolism and cancer[J]. Cell Metab. 2013;18(2):153-61.

33. Das SK, Eder S, Schauer S, et al. Adipose triglyceride lipase contributes to cancer-associated cachexia[J]. Science. 2011;333(6039):233-8.

34. Mauer J, Denson JL, Brüning JC. Versatile functions for IL-6 in metabolism and cancer[J]. Trends Immunol. 2015;36(2):92-101.

35. Ghadir MR, Riahin AA, Havaspour A, et al. The relationship between lipid profile and severity of liver damage in cirrhotic patients[J]. Hepat Mon. 2010;10(4):285.

36. Chrostek L, Supronowicz L, Panasiuk A, et al. The effect of the severity of liver cirrhosis on the level of lipids and lipoproteins[]]. Clin Exp Med. 2014; 14(4):417-21.

37. Liu X, Xiu L, Jiao J, et al. Traditional Chinese medicine integrated with chemotherapy for stage IV non-surgical gastric cancer: a retrospective clinical analysis[J]. Journal of integrative medicine. 2017;15(6):469-75

38. Sharma BR, Kim DW, Rhyu DY. Korean Chungtaejeon tea extract attenuates weight gain in C57BL/6J-Lep Ob/Ob mice and regulates adipogenesis and lipolysis in 3T3-L1 adipocytes[]]. Journal of integrative medicine. 2017:15(1):56-63.

Ready to submit your research? Choose BMC and benefit from:

- fast, convenient online submission

- thorough peer review by experienced researchers in your field

- rapid publication on acceptance

- support for research data, including large and complex data types

- gold Open Access which fosters wider collaboration and increased citations

- maximum visibility for your research: over $100 \mathrm{M}$ website views per year

At $\mathrm{BMC}$, research is always in progress.

Learn more biomedcentral.com/submissions 